\title{
A statistical framework for conditional extreme event attribution
}

\author{
Pascal Yiou $^{1}$, Aglaé Jézéquel ${ }^{1}$, Philippe Naveau ${ }^{1}$, Frederike E. L. Otto ${ }^{2}$, Robert Vautard ${ }^{1}$, and \\ Mathieu Vrac ${ }^{1}$ \\ ${ }^{1}$ Laboratoire des Sciences du Climat et de l'Environnement, UMR 8212 CEA-CNRS-UVSQ, U Paris-Saclay, \\ IPSL, Gif-sur-Yvette, France \\ ${ }^{2}$ Environmental Change Institute, School of Geography and the Environment, University of Oxford, \\ Oxford, UK
}

Correspondence to: Pascal Yiou (pascal.yiou@1sce.ipsl.fr)

Received: 13 September 2016 - Revised: 6 March 2017 - Accepted: 14 March 2017 - Published: 18 April 2017

\begin{abstract}
The goal of the attribution of individual events is to estimate whether and to what extent the probability of an extreme climate event evolves when external conditions (e.g., due to anthropogenic forcings) change. Many types of climate extremes are linked to the variability of the large-scale atmospheric circulation. It is hence essential to decipher the roles of atmospheric variability and increasing mean temperature in the change of probabilities of extremes. It is also crucial to define a background state (or counterfactual) to which recent observations are compared. In this paper we present a statistical framework to determine the dynamical (linked to the atmospheric circulation) and thermodynamical (linked to slow forcings) contributions to the probability of extreme climate events. We illustrate this methodology on a record precipitation event that hit southern United Kingdom in January 2014. We compare possibilities for the creation of two states (or "worlds") in which probability change is determined. These two worlds are defined in a large ensemble of atmospheric model simulations (Weather@Home factual and counterfactual simulations) and separate periods (new: 1951-2014, and old: 19001950) in reanalyses and observations. We discuss how the atmospheric circulation conditioning can affect the interpretation of extreme event attribution. We eventually show the qualitative coherence of results between the choice of worlds (factual/counterfactual vs. new/old).
\end{abstract}

\section{Introduction}

Many extreme events that occur on a local scale are specific to large-scale atmospheric patterns (e.g., rainfall, windstorms, heatwaves in Europe, and phases of the North Atlantic Oscillation). If such links have been identified, changes in the probability of local extremes can be due to changes in the properties of the atmospheric circulation or changes in the link between the local variable and the circulation (which can remain unchanged). The first cause is sometimes qualified as "dynamic" because it refers to the motion of the atmosphere. The second cause is qualified as "thermodynamic" (or "non-dynamic"), because it implicitly assumes that the local variable is related to the local change of atmospheric physical properties (e.g., temperature, water content) in the absence of flow changes (Trenberth et al., 2015).
The extreme event attribution (EEA) consists of estimating if and how the probability of an extreme event depends on the climate forcings (National Academies of Sciences Engineering and Medicine, 2016). One of the outcomes is the assessment of whether anthropogenic forcings alter such probability. This type of study has been used for estimates of liability for extreme events that caused damages (Allen, 2003).

The first scientific challenge of EEA is to define two worlds to be compared. The EEA studies speak of a factual world when all climate forcings (natural and anthropogenic) are considered (Stott et al., 2004; Pall et al., 2011). This is presumably a world that "is", and in which an event is observed with probability $p_{1}$. The counterfactual world contains only natural forcings, and is a world that "might have been" without anthropogenic forcings. In such a world, the same class of extreme event would occur with probability 
$p_{0}$. Defining a counterfactual world is a difficult task because it is a possible but non-observed state of climate. Then, some studies define the fraction of attributable risk (FAR), which is the relative change of probability between the two worlds FAR $\equiv\left(p_{1}-p_{0}\right) / p_{1}=1-p_{0} / p_{1}$ (Stott et al., 2004). Other combinations of the $p_{0}$ and $p_{1}$ probabilities also provide pieces of valuable information (Hannart et al., 2016) in the framework of causality theory (Pearl, 2009). The FAR is interpreted in terms of a probability of necessary causation. A probability of sufficient causation is defined by $1-\frac{1-p_{1}}{1-p_{0}}$.

An alternative approach to factual/counterfactual worlds can be proposed, as in van Haren et al. (2013): a "new" world in which we live, like the recent decades, and an "old" world in which our ancestors lived, like the beginning of the 20th century. We implicitly assume that these two worlds are different (at least from the environmental point of view). For instance, the anthropogenic forcings are likely to be stronger in the new world than in the old world. The main feature of this approach is that it can be based on observed data. It is difficult to decipher the natural and anthropogenic forcings between old and new. Moreover, the old world might not be free of anthropogenic forcings. It is just assumed that the old world is less affected than the new world by anthropogenic forcings. Therefore, such an observation-based approach can only provide qualitative information on EEA, from implicit hypotheses in the forcing changes, like "greenhouse gas forcing" is larger in the new world than in the old world.

Each of these two approaches can be summarized in terms of a universe containing two worlds (factual/counterfactual or new/old) in which probabilities of extreme events are determined.

A second challenge is to determine the dynamical and thermodynamical contributions to the change of probabilities of a class of events. We assume that extreme values of a climate variable are generally reached for given patterns of atmospheric circulation. The challenge is (i) to estimate the contribution of atmospheric variability in climate change, and (ii) to determine how the properties of a local climate variable would change if the atmospheric circulation is fixed to these patterns but forcings (natural vs. anthropogenic) are different. This is advocated by a "storyline" approach to describe a class of extreme events, by understanding the general synoptic conditions leading to the extremes (Trenberth et al., 2015; Shepherd, 2016). The storyline approach is designed to decompose the role of climate change in the dynamical and thermodynamical contributions. From a statistical point of view, this motivates the term "conditional attribution"; we investigate how the probability of a local extreme event that depends on a large-scale atmospheric circulation is affected by global climate change or the properties of the circulation itself. If we focus on precipitation extremes, the issue is to evaluate changes in atmospheric flows leading to high precipitation (the dynamical contribution) and changes in precipitation rates given a favorable atmospheric flow (the conditional thermodynamical contribution) (Trenberth et al., 2015). This requires one to define a metric to follow the atmospheric circulation conditioning. We propose two choices of such metrics and evaluate how they affect the interpretation of extreme event attribution.

The primary goal of this paper is to propose a statistical Bayesian framework to identify dynamical and thermodynamical contributions to a change of probability of a class of extreme events involving the atmospheric circulation. The Bayesian aspect emphasizes the role of atmospheric circulation trajectories that drive extreme events. For illustration purposes, we focus on the heavy precipitation event that occurred in Europe in January 2014, which has been investigated by many authors (Huntingford et al., 2014; Matthews et al., 2014; Christidis and Stott, 2015; Schaller et al., 2016). This event was a record precipitation in southern UK and Brittany (France). We test this statistical framework on a combination of two universes (factual/counterfactual and new/old) and two atmospheric circulation metrics. These four experiments allow for a focused discussion on the interpretation of extreme event attribution.

Section 2 details the datasets that are used to define two worlds. Section 3 explains the notation and methodology that is developed in the paper. Section 4 gives the results of the analyses from the two datasets. The results are discussed in Sect. 5 and conclusions appear in Sect. 6.

\section{Data}

This section explains the two universes that are considered in this study. The first one is based on a large ensemble of climate simulations. The second is based on reanalyses and observations.

\subsection{Weather@Home}

We used an ensemble of atmospheric model simulations from Weather@Home to test factual vs. counterfactual worlds. The Weather@Home data come from the "weather@home" citizen-science project (Massey et al., 2015). This project uses spare CPU time on volunteers' personal computers to run the regional climate model (RCM) HadRM3P nested in the HadAM3P atmospheric general circulation climate model (AGCM) (Massey et al., 2015) driven with prescribed sea surface temperatures (SSTs) and sea ice concentration (SIC). The RCM covers Europe and the eastern North Atlantic Ocean, at a spatial resolution of about $50 \mathrm{~km}$. These simulations were used by Huntingford et al. (2014) and Schaller et al. (2016) to investigate the impact of climate change on the extreme precipitation of January 2014 in southern UK.

The factual world is made of $\approx 17000$ winters (December-January-February: DJF) simulated under observed 2013/2014 greenhouse gas (GHG) concentrations, SSTs and SICs. Initial conditions are perturbed slightly for 
each ensemble member on 1 December to give a different realization of the winter weather.

The counterfactual world is made of $\approx 117000$ simulations with different estimates of conditions that might have occurred in a world without past emissions of GHGs and other pollutants including sulfate aerosol precursors. The atmospheric composition is set to the pre-industrial, the maximum well-observed SIC is used (DJF 1986/1987) and estimated anthropogenic SST change patterns are removed from observed DJF 2013/2014 SSTs (Schaller et al., 2016). To account for the uncertainty in the estimates of a world without anthropogenic influence, 11 different patterns are calculated from climate model simulations of the Coupled Model Intercomparison Project phase 5 (CMIP5) (Taylor et al., 2012).

The circulation $C$ is taken from the sea-level pressure (SLP) data of the RCM simulations. The climate variable $R$ is the southern UK precipitation averaged over land grid points in $50-52^{\circ} \mathrm{N}, 6.5^{\circ} \mathrm{W}-2^{\circ} \mathrm{E}$. Simulated $R$ for the factual ensemble members with the wettest $1 \%$ are comparable to observations of January 2014. The mean climate of the RCM has a wet (positive) bias of $+0.4 \mathrm{~mm} \mathrm{day}^{-1}$ in January over southern UK (Schaller et al., 2016) but most RCM simulations for January 2014 show smaller anomalies than in the observations reported by Matthews et al. (2014), and show a weaker SLP pattern for the same precipitation anomaly. On average, the factual simulations reproduce a stronger jet stream, compared to the 1986-2011 climatology of January 2014 in the North Atlantic, suggesting some potential predictability for the enhanced jet stream of January 2014 (Schaller et al., 2016). The differences in SSTs, SICs and atmospheric composition between the two sets of simulations lead to an increase (from the counterfactual to factual) of up to $0.5 \mathrm{~mm} \mathrm{day}^{-1}$ in the wettest $1 \%$ ensemble members for January southern United Kingdom precipitation.

\subsection{Reanalyses and observations}

The comparison of old vs. new worlds was performed with two reanalysis datasets. We consider the circulation $C$ from the SLP over the North Atlantic region $\left(80^{\circ} \mathrm{W}-50^{\circ} \mathrm{E} ; 25-\right.$ $70^{\circ} \mathrm{N}$ ) for both reanalyses. The new world is made of the National Centers for Environmental Prediction (NCEP) reanalysis data for the winters (December to February) between 1951 and 2014 (Kalnay et al., 1996). The old world is made of the 20CR reanalysis dataset for the winters between 1900 and 1950 (Compo et al., 2011). The reason why both reanalyses need to be considered is that $20 \mathrm{CR}$ ends in 2011 and hence does not include the winter 2013/2014, in which we are interested, for the case study of Schaller et al. (2016). A few tests on the statistical properties of the circulation in both reanalyses were performed on their overlapping period (Schaller et al., 2016). It appears that in spite of using different climate models and with different resolutions, both reanalyses exhibit similar features. This means that the

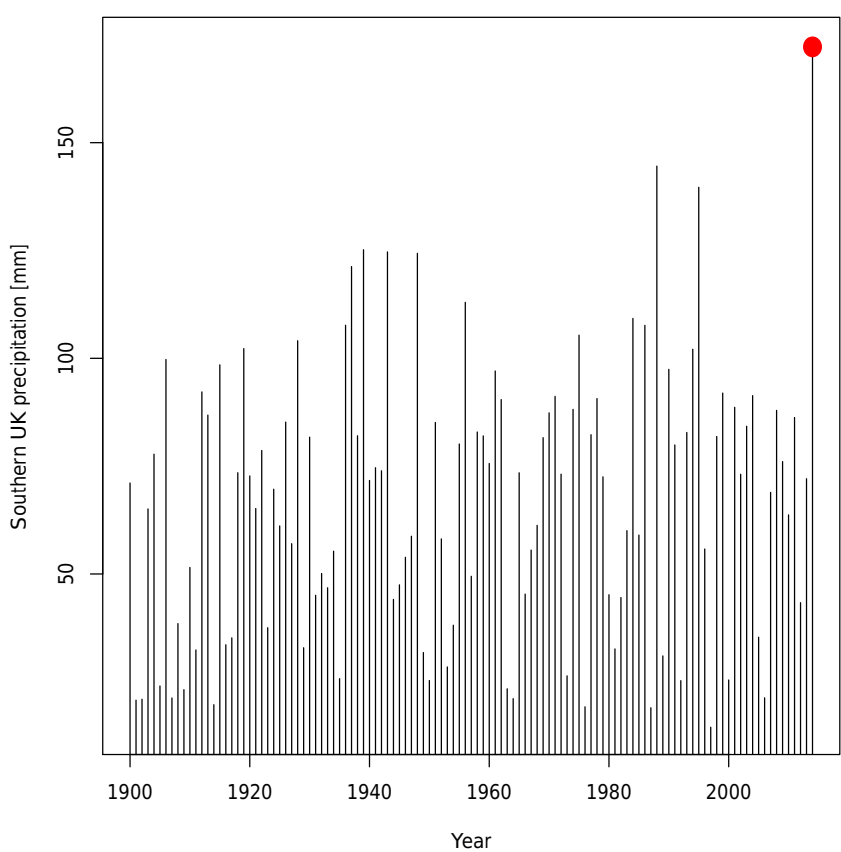

Figure 1. Time series of January cumulated observed precipitation in southern United Kingdom between 1900 and 2014 (in mm). The red dot indicates the value of $R$ for January 2014 .

shift from the old world (with 20CR) to the new world (with NCEP) is rather smooth.

The precipitation $R$ is taken from daily precipitation observations from the UK Met Office (Matthews et al., 2014) between 1900 and 2014. The dataset consists of observations from 14 stations in the southern UK. These stations include Oxford, Rothamsted, Wisley, Bognor Regis, Cambridge, Eastbourne, East Malling, Goudhurst, Hampstead, Hampton, Larkhill, Otterbourne, Shanklin (Isle of White) and Woburn. The variable $R$ is an average of daily values of these 14 stations. We verify that a record of January monthly precipitation was reached in 2014 (Fig. 1).

\section{Methodology}

\subsection{Notations and rationale}

We assume that a climate variable $R$ (e.g., temperature, precipitation) and atmospheric circulation $C$ (e.g., SLP, geopotential height at $500 \mathrm{hPa}$ ) are observed in a universe that contains two distinct worlds that we call $\mathcal{W}_{0}$ and $\mathcal{W}_{1}$. Here, $R$ is a real variable and $C$ is a two-dimensional field. For the first universe, $\mathcal{W}_{1}$ is the "factual" world and $\mathcal{W}_{0}$ is the "counterfactual" world. This universe is represented by the Weather@Home ensemble. In the second universe $\mathcal{W}_{1}$ is the new world and $\mathcal{W}_{0}$ is the old world. This universe is represented by the NCEP (1951 to 2014) and 20CR (1951 to 2014) reanalyses, and observed precipitation. We specify in 
the text the universes to which the worlds $\mathcal{W}_{i}$ belong, in order to avoid unnecessarily complicated notations.

We recall that the $\mathcal{W}_{1}$ worlds (in the two universes) are close to the one in which we live, either in terms of anthropogenic/natural climate forcings or in terms of temporal proximity (e.g., the last decades). The $\mathcal{W}_{0}$ worlds contain only natural climate forcings, or temporal remoteness (e.g., beginning of 20th century: 1900-1950 vs. recent decades: 1951-2014).

We define an extreme event (in either worlds and universes) when a reference threshold $R_{\text {ref }}$ for $R$ has been equalled or exceeded. A "class of events" includes the ensemble of weather types for which the threshold can be equalled or exceeded. In this paper, we assume that such an extreme event is reached during a spell of atmospheric circulation $C_{\text {ref }}$ in the world $\mathcal{W}_{1}$.

The goal of extreme event attribution is to determine how the probability of an extreme event differs between $\mathcal{W}_{1}$ and $\mathcal{W}_{0}$. Achieving this goal is trivial if a rare event can occur in one of the worlds and cannot in the other. In practice, this does not happen for most extreme events that have occurred in the past decades, because there are often historical examples of such events (e.g., most European winter storms, European heatwaves). Thus, we assume that a given extreme or rare climate event has a probability of occurrence $p_{1}$ in $\mathcal{W}_{1}$, and $p_{0}$ in $\mathcal{W}_{0}$.

The probabilities $p_{1}$ and $p_{0}$ are defined by

$p_{i}=\operatorname{Pr}\left(R_{(i)}>R_{\text {ref }}\right)$,

where $R_{(i)}$ is the climate variable $R$ in the $\mathcal{W}_{i}$ world, and $i \in\{0,1\}$.

For obvious pragmatic reasons, we can assume that $p_{1}>$ 0 , because we want to study an event that was observed in the real world. In addition, $p_{1}$ can be fixed to a quantile of the probability distribution of $R$ in $\mathcal{W}_{1}$. Here we take $p_{1}=0.01$ to be consistent with (Schaller et al., 2016). This could be interpreted in a one-in-a-century event if the data have a yearly sampling. This defines a class of events (here high values of $R$ ). Therefore, there is no uncertainty in the determination of $p_{1}$. The uncertainty is shifted to the estimate of $R_{\text {ref }}$ from $\mathcal{W}_{1}$ data (if $1 / p_{1}$ is larger than the size of $\mathcal{W}_{1}$ ), and in $p_{0}$.

We want to estimate the ratio $p_{0} / p_{1}$, determine its uncertainty and investigate how it is controlled by physical factors. These physical factors include changes in the probability distribution of the circulation $C$ between $\mathcal{W}_{1}$ and $\mathcal{W}_{0}$ and the changes in the probability distribution of $R$ if $C$ is similar in $\mathcal{W}_{1}$ and $\mathcal{W}_{0}$. We introduce the notion of vicinity of circulation trajectories, or the neighborhood $\mathcal{V}$ of an observed circulation $C_{\text {ref. }}$. The trajectory neighborhood will be defined in two ways: from the distance to a known weather regime (Sect. 3.3.1), which is computed independently of the event itself, or from the distance to the observed trajectory of circulation (Sect. 3.3.2).

\subsection{A conditional formulation of extreme event attribution}

The probabilities $p_{i}(i \in\{0,1\})$, which represent the marginal probability that the climate variable $R_{(i)}$ exceeds a threshold $R_{\text {ref }}$ (unconditional on the circulation) in world $\mathcal{W}_{i}$, can be decomposed into a product of conditional probabilities involving the atmospheric circulation $C_{(i)} \in \mathcal{V}\left(C_{\text {ref }}\right)$ using rules of probability (Bayes' formula) as follows:

$$
\begin{aligned}
p_{i} \equiv \operatorname{Pr}\left(R_{(i)}>R_{\mathrm{ref}}\right) \quad & \operatorname{Pr}\left(R_{(i)}>R_{\mathrm{ref}} \mid C_{(i)} \in \mathcal{V}\left(C_{\mathrm{ref}}\right)\right) \\
& \times \operatorname{Pr}\left(C_{(i)} \in \mathcal{V}\left(C_{\mathrm{ref}}\right)\right) \\
& / \operatorname{Pr}\left(C_{(i)} \in \mathcal{V}\left(C_{\mathrm{ref}}\right) \mid R_{(i)}>R_{\mathrm{ref}}\right)
\end{aligned}
$$

The three terms of the right-hand side of Eq. (2) can be computed from data in the two worlds $\mathcal{W}_{i}$.

The ratio $\rho=p_{0} / p_{1}$ is then decomposed into three terms that can yield physical interpretations. The first one is the thermodynamical change between the two worlds for a given circulation:

$\rho^{\text {the }} \equiv \frac{\operatorname{Pr}\left(R_{(0)}>R_{\text {ref }} \mid C_{(0)} \in \mathcal{V}\left(C_{\text {ref }}\right)\right)}{\operatorname{Pr}\left(R_{(1)}>R_{\text {ref }} \mid C_{(1)} \in \mathcal{V}\left(C_{\text {ref }}\right)\right)}$.

In this term, the circulation is fixed to one that is close to $C_{\text {ref }}$, and changes of the probability of $R$ are due to causes such as an increased temperature (increasing the water availability in the atmosphere, Peixoto and Oort, 1992). If the $C_{\text {ref }}$ pattern is prone to high precipitation, this conditional term allows for a closer focus on the tail of the distribution of $R$.

The second term accounts for changes in the patterns of the atmospheric circulation and is hence called "circulation":

$\rho^{\mathrm{circ}} \equiv \frac{\operatorname{Pr}\left(C_{(0)} \in \mathcal{V}\left(C_{\mathrm{ref}}\right)\right)}{\operatorname{Pr}\left(C_{(1)} \in \mathcal{V}\left(C_{\mathrm{ref}}\right)\right)}$

It is important to note that $C_{\text {ref }}$ is the same in the numerator and denominator. The circulation term measures the change of likelihood of observing circulation sequences that look like $C_{\text {ref. }}$.

The third term is a reciprocity condition for the circulation trajectory $C$ :

$\rho^{\mathrm{rec}} \equiv \frac{\operatorname{Pr}\left(C_{(1)} \in \mathcal{V}\left(C_{\mathrm{ref}}\right) \mid R_{(1)}>R_{\mathrm{ref}}\right)}{\operatorname{Pr}\left(C_{(0)} \in \mathcal{V}\left(C_{\mathrm{ref}}\right) \mid R_{(0)}>R_{\mathrm{ref}}\right)}$.

This term determines the extent to which the circulation $C_{\text {ref }}$ is necessary when $R>R_{\text {ref }}$. For a fixed $R_{\text {ref precipita- }}$ tion rate, it evaluates how likely a circulation such as $C_{\text {ref }}$ is. This reciprocity term allows one to connect the risk-based approach of EEA, based on the study of $\rho$ alone (Shepherd, 2016) to the "storyline approach" (Trenberth et al., 2015; National Academies of Sciences Engineering and Medicine, 2016), which involves the processes that drive the extreme precipitation.

The product $\rho^{\text {dyn }} \equiv \rho^{\text {circ }} \times \rho^{\text {rec }}$ defines the dynamical contribution of the atmospheric change to the precipitation extreme conditional to a fixed thermodynamics. The reciprocity 
term explores the extent to which the circulation is close to the observed one when the cumulated precipitation is high. This multiplicative decomposition of probabilities can be compared with the "additive" decomposition of Shepherd (2016, Eq. 1), who also introduces a non-dynamical term. Our decomposition allows for the fact that the probability distribution of $R$ and $C$ could remain unchanged between $\mathcal{W}_{0}$ and $\mathcal{W}_{1}$, while the physical link between these variables evolve in compensating ways; the probability of having a high $R$ when $C$ is close to $C_{\text {ref }}$ could decrease and the probability of having $C$ close to $C_{\text {ref }}$ when $R$ is high could increase.

Sampling uncertainties on these three ratios can be determined by bootstrapping over the elements of $\mathcal{W}_{i}$.

The estimation procedure is the following:

1. determine $p_{1}$ (for example a century return period) and an empirical $R_{\text {ref }}$ (for example from $\mathcal{W}_{1}$ );

2. determine the neighborhood of $C_{\text {ref }}$ (for example from the monthly frequency of a weather regime);

3. determine $\rho^{\text {the }}, \rho^{\text {circ }}, \rho^{\text {rec }}$ and their sampling distribution for the two worlds, for example by bootstrapping over $\mathcal{W}_{i}$. The bootstrap is done by repeating random samples of seasons so that the intra-seasonal coherence is preserved.

We then assess whether $\rho^{\text {the }}, \rho^{\text {circ }}$ and $\rho^{\text {rec }}$ are significantly different from 1 by comparing their sampling distributions. We denote $\bar{\rho}$ the estimate of each ratio from all data. The 5 th and 95th quantiles ( $\hat{\rho}^{5 \%}$ and $\hat{\rho}^{95 \%}$, respectively) of the bootstrap simulations provide an interval of the sampling confidence interval $\left(\bar{\rho}-\left(\hat{\rho}^{95 \%}-\bar{\rho}\right), \bar{\rho}-\left(\hat{\rho}^{5 \%}-\bar{\rho}\right)\right)$

We will illustrate this approach on the high precipitation event of the winter 2013/2014 in southern UK.

\subsection{Circulation neighborhood}

In this section, we propose two ways of defining the neighborhood of the circulation $C_{\text {ref }}$. This has an impact on the computation of the thermodynamical and dynamical terms of the decomposition of $\rho$.

\subsubsection{Proximity based on weather regimes}

High winter precipitation in Europe is generally associated with zonal atmospheric circulation. The circulation around the North Atlantic can be described by four weather regimes, which are quasi-stationary states of the atmosphere (Vautard et al., 1988; Kimoto and Ghil, 1993; Michelangeli et al., 1995). These weather regimes are obtained by a $K$ means classification of anomalies of the winter SLP daily field from the NCEP reanalysis (Michelangeli et al., 1995; Yiou et al., 2008) on a reference period (1970-2000). The weather regime centroids are shown in Fig. 2.
The weather regimes of the $20 \mathrm{CR}$ reanalysis are the same as for NCEP, as well as the regime frequencies (Schaller et al., 2016, supplementary Fig. 7). After a removal of the mean, the SLP of Weather@Home simulations is projected onto these reference centroids to compute the weather regime frequencies. This is done to ensure the consistency of the interpretation of the regime frequencies.

The frequencies of the weather regimes are computed for each winter season (December-January-February). Very wet winters in the UK or northwestern France occur when the frequencies of zonal $(\mathrm{ZO})$ or negative phase of the North Atlantic Oscillation (NAO-) weather regimes are high $(\geq 75 \%)$. This threshold duration roughly corresponds to the 97th quantile of frequency for the zonal (ZO) regime in Weather@Home simulations. This allows one to have a non-zero probability of $\operatorname{Pr}\left(C_{(i)} \in \mathcal{V}\left(C_{\text {ref }}\right)\right.$ for the $\mathrm{ZO}$ regime in both reanalysis worlds.

The average frequency of the zonal weather regime is close to $25 \%$ and the frequency reached $81 \%$ in January 2014. The two other weather regimes (Scandinavian blocking and Atlantic Ridge) do not lead to very high precipitation rates in southern UK. The zonal weather regime favors warm temperatures in Europe, while NAO- favors cold temperatures (Yiou and Nogaj, 2004; Cattiaux et al., 2010).

The atmospheric trajectories can then be tracked by daily sequences of weather regimes. We summarize the information of a trajectory over a whole winter season (or a single winter month) by the frequencies of the four weather regimes. Hence, if $C_{\text {ref }}$ was mainly zonal (as was the winter of 2013/2014), we will say that the circulation $C$ is in the neighborhood of $C_{\text {ref }}\left(C \in \mathcal{V}\left(C_{\text {ref }}\right)\right)$ if the frequency of the zonal weather regime exceeds $75 \%$. This definition obviously oversimplifies the notion of circulation neighborhood, but it gives an intuitive and qualitative understanding of the atmospheric circulation. This approach is also taken for consistency with the study of Schaller et al. (2016).

\subsubsection{Proximity based on analogues of circulation}

The computation of weather regimes provides an intuitive and physical interpretation of the atmospheric circulation patterns. But the atmospheric flow trajectories that are considered are, by construction, just closer to one of the weather regime centroids than the others, and not necessarily close to the circulation that prevailed during the event, which could be atypical in terms of weather regimes. Hence, we also explore the atmospheric circulation with analogues, which exploit explicitly a distance to a reference observed circulation pattern sequence.

If $C(d)$ is the SLP during some day $d$, the analogues of $C$ are the days $d_{k}$ in a different year, for which the Euclidean distance $d\left(C(d), C\left(d_{k}\right)\right)$ is minimized. This defines analogues of circulation, based on SLP. Here we consider the North Atlantic sector $\left(80^{\circ} \mathrm{W}-50^{\circ} \mathrm{E} ; 25-70^{\circ} \mathrm{N}\right)$ to compute the distance between two SLP patterns, as in Yiou et al. 

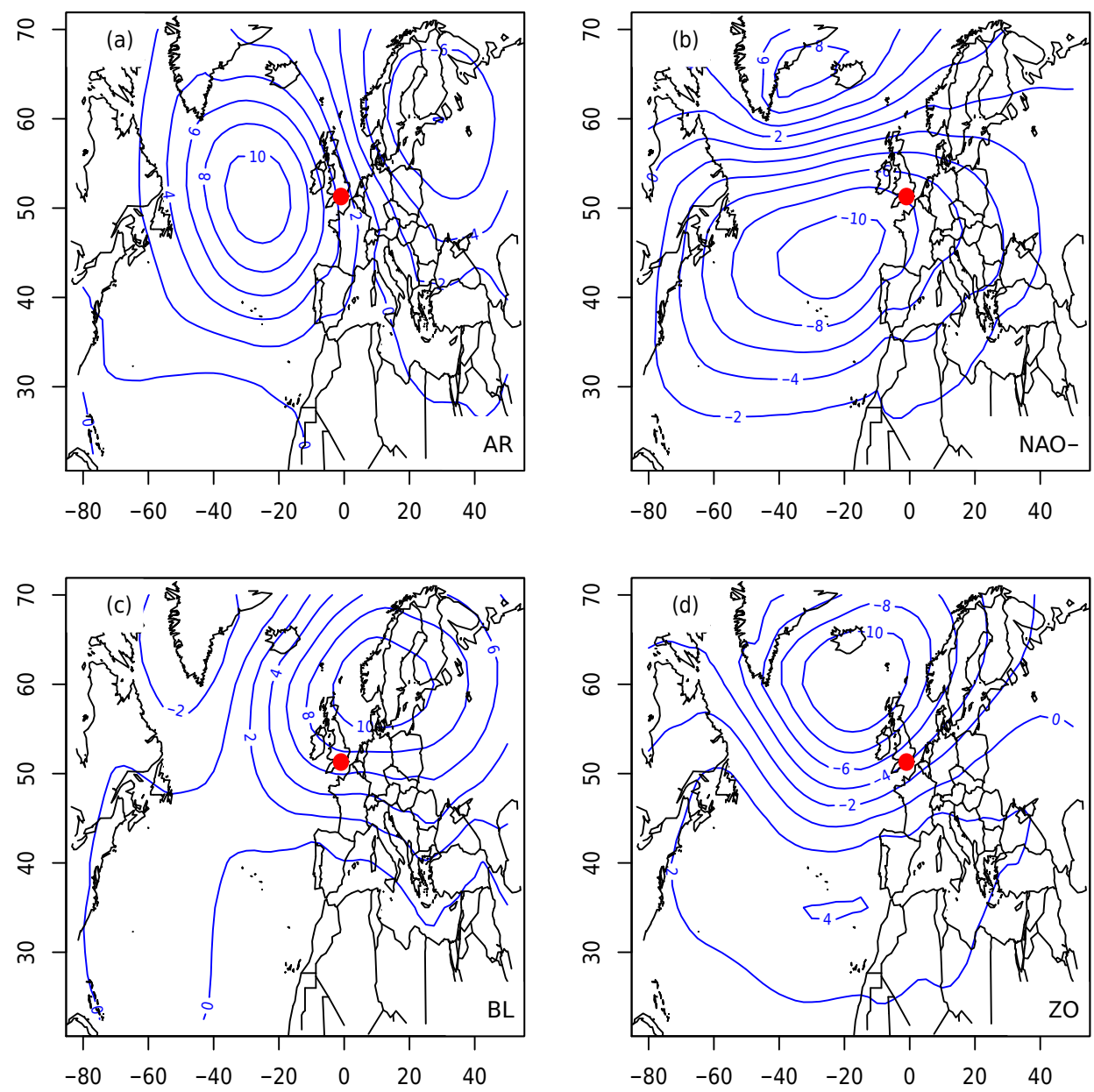

Figure 2. Four winter (DJF) weather regimes of the North Atlantic, computed from the SLP anomalies (in hPa) of NCEP reanalysis. (a) Atlantic Ridge (AR); (b) NAO-; (c) Scandinavian blocking (BLO); (d) zonal (ZO). The red circles indicate the region where high precipitation was observed.

(2013). We take the $K=20$ best analogues of circulation for each day.

A justification to use analogues of circulation to describe the January 2014 atmospheric circulation comes from the fact that the SLP had a rather unusual pattern, which did not have all the characteristics of the zonal weather regime shown in Fig. 2. We illustrate this in Fig. 3 with the mean of analogues from $\mathcal{W}_{0}$ (1900-1950 in 20CR; Fig. 3c) and $\mathcal{W}_{1}$ (1950-2014 in NCEP; Fig. 3d). The mean SLP yields a rather steep gradient over UK and France. This steep SLP gradient is better reproduced in the analogue mean than in the $\mathrm{ZO}$ weather regime.

A heuristic way to define the neighborhood of the trajectory $C_{\text {ref }}$ (e.g., a sequence of $C(d)$ with days in January 2014) is to compute the mean (over the days) of a quantile of the distances of the best analogues of $K$. This value can be modulated by a "safety" factor to ensure that there are enough trajectories around $C_{\text {ref }}$ to construct statistics. This defines a neighboring "tube" around $C_{\text {ref }}$ in the SLP phase space.
This threshold is computed from the analogues of $C_{\text {ref }}$ in January 2014 for the NCEP reanalyses (1950-2014, excluding January 2014) and gives a value of $\approx 12 \mathrm{hPa}$ for a median quantile of the $K=20$ best daily analogues and a safety factor of 1.5.

In addition to a definition of proximity, we use the dates of the best SLP analogues simulated reconstructions of climate variables. Here we focus on precipitation $R$. From a statistical perspective, the analogue precipitation is random "replicates" of the precipitation at the day conditioned by the atmospheric circulation. This allows for a determination of the probability distributions of precipitation $(R)$ variability conditioned to the atmospheric circulation $C$.

Analogues of $C$ and $R$ provide a natural way of computing the probabilities in Eq. (2). We compute this estimate from the reanalysis datasets $\left(\mathcal{W}_{0}=20 \mathrm{CR}\right.$ and $\left.\mathcal{W}_{1}=\mathrm{NCEP}\right)$. By contrast, we test the null hypothesis $\mathrm{H} 0$ that circulation does not play a role in the high precipitation rate by computing the probability distribution of cumulated precipitation 

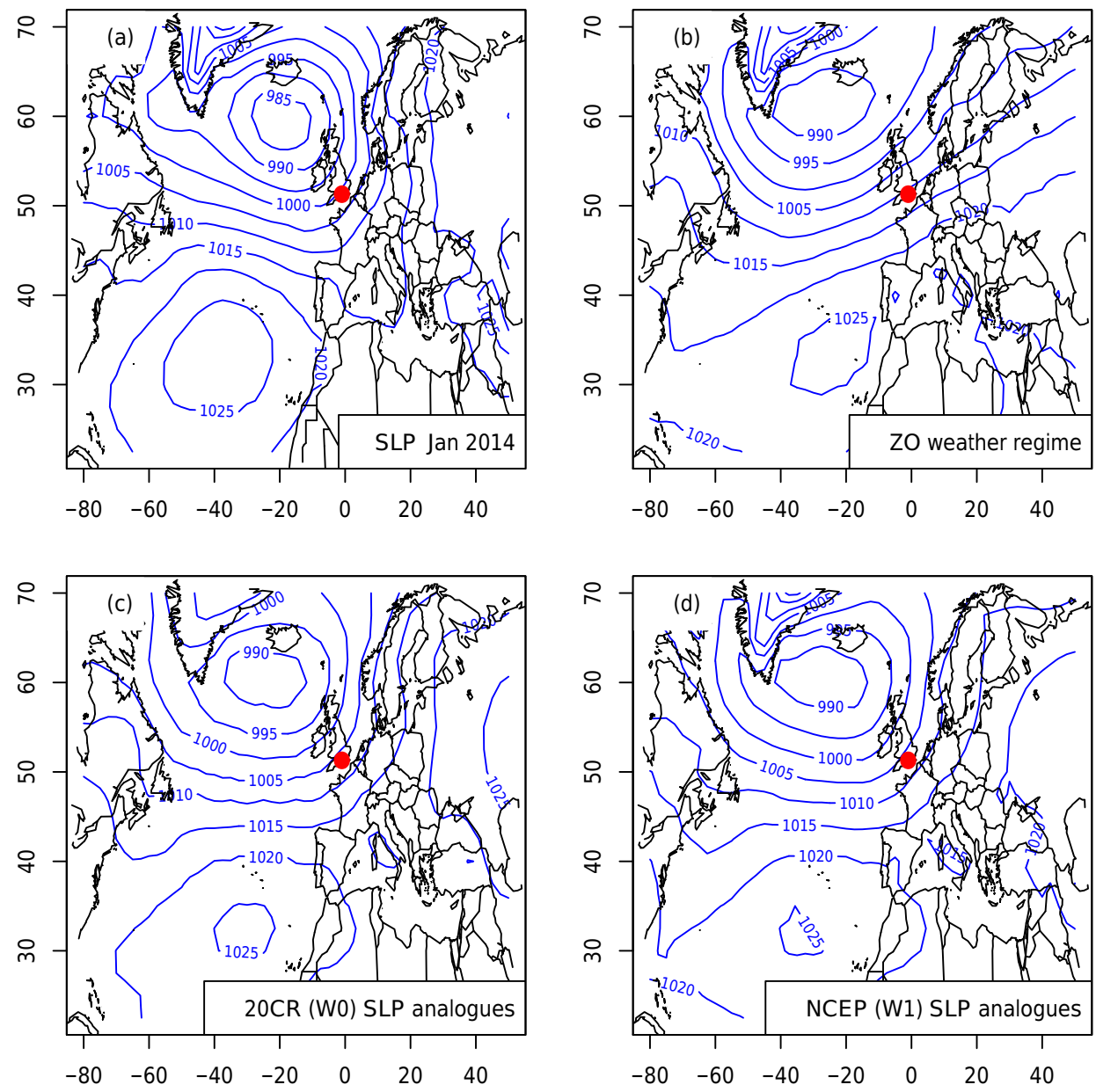

Figure 3. The mean SLP of January 2014 (in hPa) for (a) NCEP reanalysis (b) ZO weather regime computed from NCEP (Fig. 2d); (c) Mean of analogues in 20CR $\left(1900-1950, \mathcal{W}_{0}\right)$; (d) Mean of analogues in NCEP $\left(1950-2014, \mathcal{W}_{1}\right)$. The red circles indicate the region where high precipitation was observed.

in January when random days are drawn in $\mathcal{W}_{0}=20 \mathrm{CR}$ and $\mathcal{W}_{1}=$ NCEP. Hence, the null hypothesis $\mathrm{H} 0$ provides an estimate of the probability distribution of cumulated random precipitation for January months. We use a KolmogorovSmirnov test (von Storch and Zwiers, 2001, p. 81) to examine the difference between the $\mathrm{H} 0$ distribution and the circulation-dependent precipitation distribution. We decide to reject $\mathrm{H} 0$ at the $1 \%$ level. When comparing the first and second (and third and fourth) box plots, Fig. 4 emphasizes the rejection of this null hypothesis because the distribution of analogue cumulated precipitation probabilities are significantly higher than for random days. In both cases (NCEP and 20CR), $\mathrm{H} 0$ is rejected with a level far below $1 \%$.

The $\rho$ term is estimated by random resampling of daily $R$ values in January and computing a monthly average. The probability distribution simulations of $R$ in January 2014 for circulation analogues in $\mathcal{W}_{0}=20 \mathrm{CR}$ and $\mathcal{W}_{1}=$ NCEP are shown in Fig. 4. For comparison purposes, mean precipitation taken from random days in the two worlds are also shown, to emphasize the role of the circulation in the high precipitation event in January. By comparing the second and fourth box plots, Fig. 4 shows a slight increase of the probability of having high precipitation in the new world with respect to the old world. The uncertainty on $\rho$ can be estimated from these box plots.

The thermodynamical term is estimated from probabilities of $R$ for analogues of $C_{\text {ref }}$ in $\mathcal{W}_{1}$ and $\mathcal{W}_{0}$. The first step is to compute analogues of $C_{\text {ref }}$ (the circulation in January 2014) in the two reanalysis datasets. For each day $d$ of January 2014, we draw random circulation analogues in $\mathcal{W}_{1}$ and $\mathcal{W}_{0}$, and keep the sequence of their dates. Then we compute the sum of the analogue $R$ for January 2014. By repeating this procedure, we obtain a Monte-Carlo estimate of the probability distributions of $R>R_{\text {ref }}$ conditional to $C_{\text {ref }}$ for the old and new worlds. This procedure is similar to the static weather generator based on analogues described by Yiou (2014). This procedure allows one to estimate the probabil- 


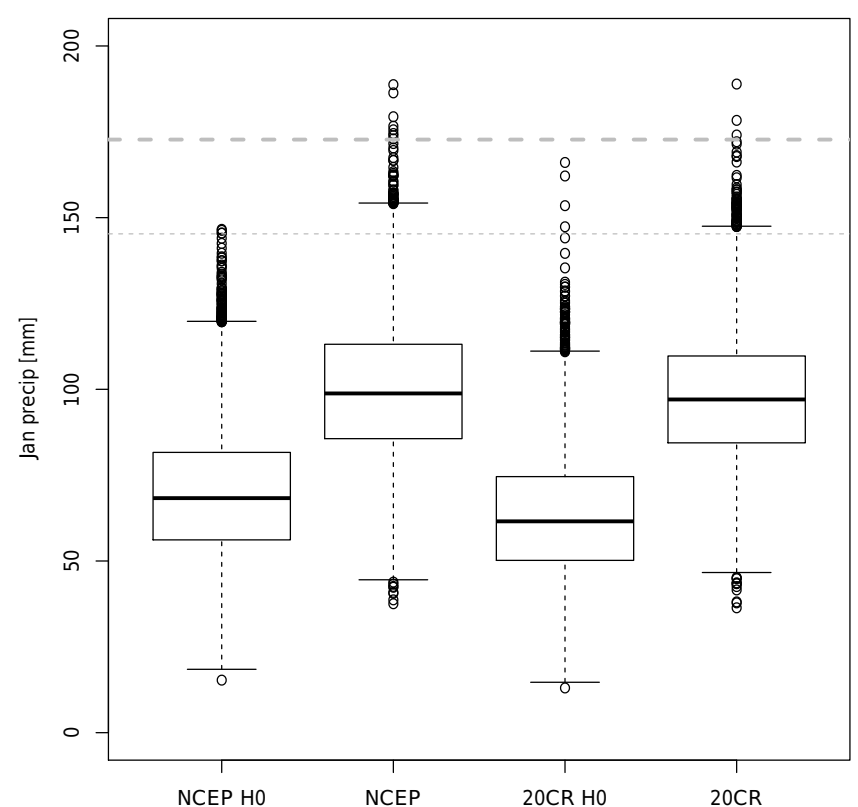

Figure 4. Box plots of cumulated precipitation simulations (in mm month ${ }^{-1}$ ) from circulation analogues of January 2014 from 20CR (1900-1950) and NCEP (1951-2014). The NCEP H0 and 20CR H0 box plots of precipitation are taken from random days in January in 20CR and NCEP (rather than analogues). The horizontal thick dashed line is the observed value for January 2014. The horizontal thin dashed line is the 99th quantile of DJF monthly precipitation. The box plot lines indicate the 25 th $\left(q_{25}\right)$, median $\left(q_{50}\right)$ and 75th $\left(q_{75}\right)$ quantile (boxes). The upper whiskers classically indicate $\min \left(1.5 \times\left(q_{75}-q_{25}\right)+q_{50}, \max (R)\right)$. The lower whiskers have a conjugate formula for low values.

ity distribution of $\rho^{\text {the }}$. In this study, we produce $N=1000$ random samples of $C$ and corresponding $R$.

The dynamical term $\rho^{\text {dyn }}$ is obtained by dividing $\rho$ by $\rho^{\text {the }}$ (and using the Bayes formula). This procedure does not give an easy access to the circulation and reciprocity terms because it samples the vicinity of $C_{\text {ref }}$, not all the possible trajectories of SLP, including those which are not close to $C_{\text {ref. }}$.

\section{Results}

\subsection{Weather@Home}

The daily SLP anomalies of the model simulations were classified onto the NCEP reanalysis weather regimes of Fig. 2. For each month, the four weather regime frequencies were computed.

For simplification we pooled all $\mathcal{W}_{0}$ simulations, unlike Schaller et al. (2016), who investigated each ensemble of counterfactual simulations separately. For each of the weather regimes (Atlantic Ridge: AR; zonal: ZO; NAO-; Scandinavian blocking: BLO), we determined the conditional probability distribution of January precipitation in southern UK when a weather regime frequency exceeds $75 \%$
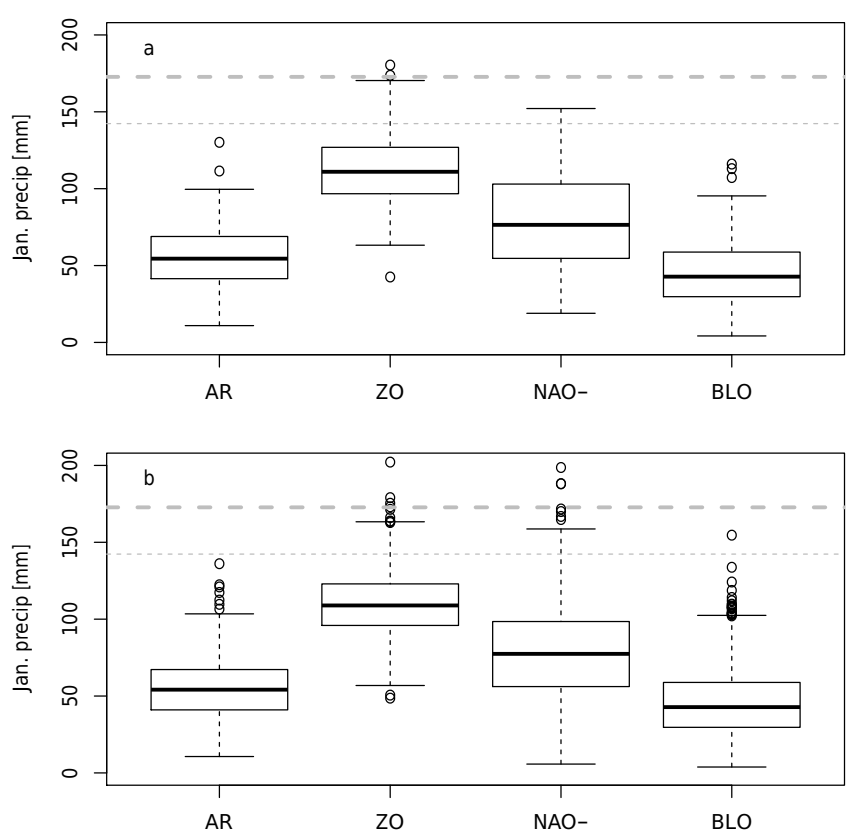

Figure 5. January precipitation probability distribution (box plots) conditional to winter weather regimes exceeding $75 \%$ in Weather@Home simulations (a: $\mathcal{W}_{1}$ factual world; $\mathbf{b}: \mathcal{W}_{0}$ counterfactual world). The thin dashed horizontal line is the $99 \%$ quantile of the $\mathcal{W}_{1}$ (factual) Weather@Home simulations. The thick dashed horizontal line is the observed precipitation value for January 2014.

of the month. Figure 5 shows that only $\mathrm{ZO}$ and NAOweather regimes reach the record values observed in January 2014, for $\mathcal{W}_{0}$ and $\mathcal{W}_{1}$. A dominant zonal weather regime obviously increases the probability of high precipitation in the winter, although extreme precipitation can also be reached with the NAO- pattern. A visual comparison of the two panels of Fig. 5 suggests that the probability of exceeding the 99th precipitation quantile in $\mathcal{W}_{1}$ slightly increases from $\mathcal{W}_{0}$ to $\mathcal{W}_{1}$, because the upper whiskers of the box plots increase. This visual impression is quantified by the analysis proposed in Sect. 3.2. The fact that precipitation can reach higher values in the counter factual world (Fig. 5b) is due to the fact that $\mathcal{W}_{0}$ contains approximately 7 times more simulations than $\mathcal{W}_{1}$.

Figure 5 shows that the North Atlantic circulation patterns are discriminating for heavy precipitation in southern UK. Hence, we focus on the zonal and NAO- atmospheric patterns to compute the probability changes.

The difference of high precipitation distribution between $\mathcal{W}_{0}$ and $\mathcal{W}_{0}$ is determined by quantile-quantile plots for each weather regimes (Fig. 5). This quantile-quantile plot can only be obtained for a large ensemble such as Weather@Home, which effectively sample persisting atmospheric patterns and high precipitation. Such a diagram cannot be obtained for observations, which do not yield a sufficient number of data over the 20th century. 

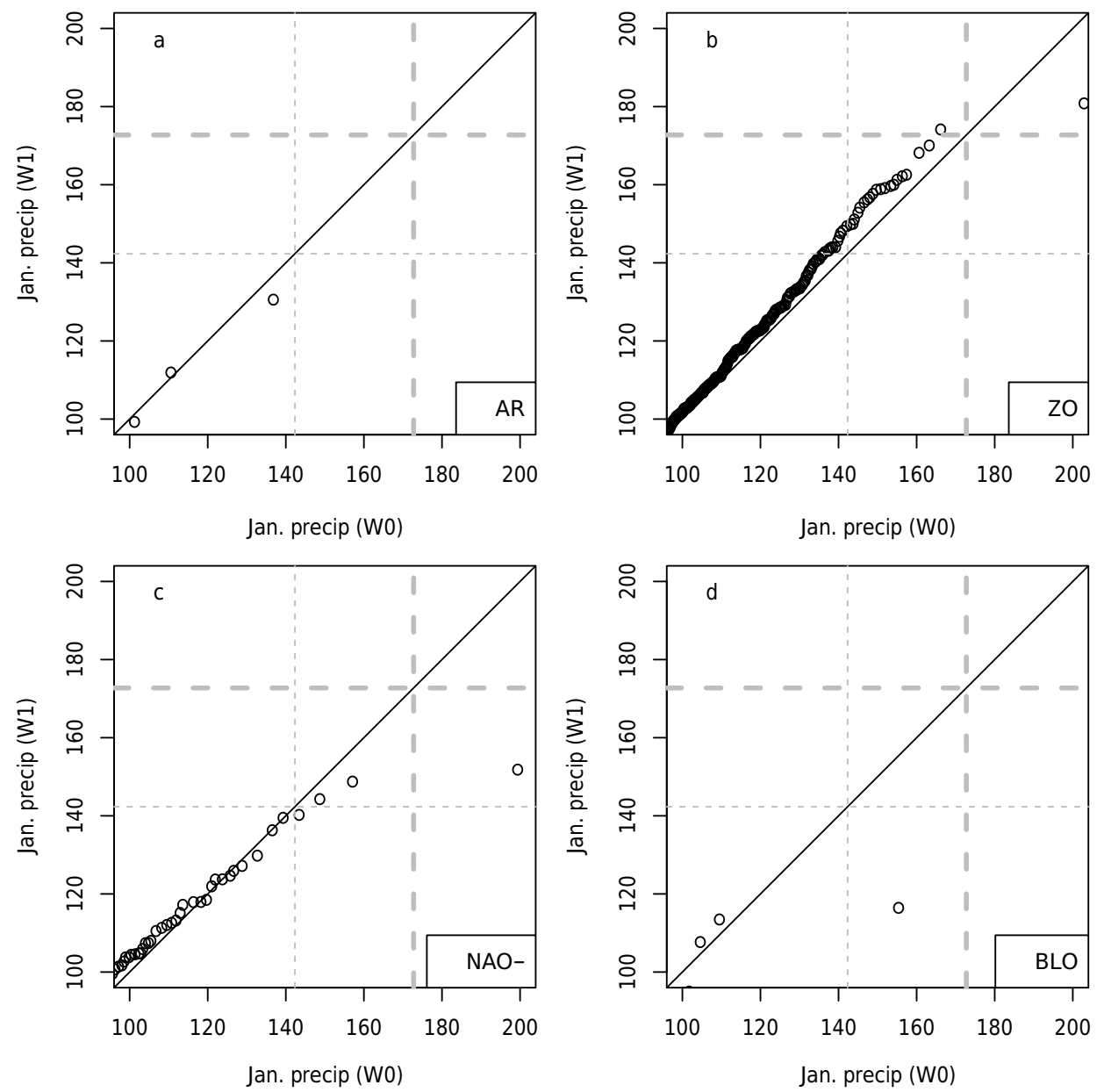

Figure 6. Quantile-quantile plots of January precipitation (in mm month ${ }^{-1}$ ) probability distributions between counterfactual $\left(\mathcal{W}_{0}\right)$ and factual $\left(\mathcal{W}_{1}\right)$ worlds in Weather@Home simulations, for each weather regime (a: Atlantic Ridge; b: zonal; c: NAO-; d: Scandinavian blocking). The continuous line is the first diagonal. The thick dashed lines indicate the observation in January 2014. The thin dashed line indicates the 99th quantile of observed January precipitation.

Figure $6 \mathrm{~b}$ shows that $\mathcal{W}_{1}$ simulations are generally wetter than $\mathcal{W}_{1}$ for the zonal weather regime, apart from one extreme exception. The precipitation distributions are rather similar for the NAO - weather regime, albeit for an extreme value that far exceeds the observed record (Fig. 6c). The two weather regimes (Atlantic Ridge and Scandinavian blocking) hardly reach the value of the 99th quantile of observed precipitation. Figure 6 hence justifies a posteriori our methodology to compare the tails of the distributions of precipitation totals. The remainder of the paper focuses on the circulation patterns for which precipitation is likely to exceed the 99th quantile of observations.

The $\rho$ ratios were computed from the $(\approx 17000)$ factual and $(\approx 117000)$ counterfactual Weather@Home simulations. Since $p_{1}$ is fixed to be 0.01 (for a return period of 1 century), the spread of $\rho$ stems from the uncertainty on $p_{0}$ that is computed over the pooled counterfactual simulations (although, strictly speaking, $R_{\text {ref }}$ uncertainty depends on the bootstrap sample from $\mathcal{W}_{1}$ ). The distribution of $\rho$ is significantly different from 1 , with a mean value $\bar{\rho}=0.71$ (Fig. 7, "all" box plot). This indicates an increase of the probability of heavy precipitation in $\mathcal{W}_{1}$ with respect to $\mathcal{W}_{0}$, with a fraction of attributable risk $\left(\mathrm{FAR}=1-p_{0} / p_{1}\right)$ of 0.29 . This probability ratio can be decomposed for the $\mathrm{ZO}$ and NAOweather regimes. The estimates of $\rho^{\text {the }}, \rho^{\text {circ }}$ and $\rho^{\text {rec }}$ for the $\mathrm{ZO}$ and NAO- weather regimes are shown in Fig. 7. By construction, the products of the mean values recover the mean value of $\rho$ (all box plot).

The three mean ratios $\left(\bar{\rho}^{\text {the }}, \bar{\rho}^{\text {circ }}\right.$ and $\left.\bar{\rho}^{\text {rec }}\right)$ are significantly different from 1 for the zonal regime $\left(\bar{\rho}^{\text {the }} \approx 0.63\right.$, $\bar{\rho}^{\text {circ }} \approx 0.78$ and $\bar{\rho}^{\text {rec }} \approx 1.45$ ). The $\rho^{\text {the }}<1$ is interpreted by an increase of precipitation from $\mathcal{W}_{0}$ to $\mathcal{W}_{1}$ given the same weather regime flow. $\rho^{\text {circ }}<1$ reflects an increase of the frequency of zonal patterns in $\mathcal{W}_{1}$ with respect to $\mathcal{W}_{0}$. $\rho^{\text {rec }}>1$ reflects that large precipitation amounts occur more often during episodes of zonal circulation. 

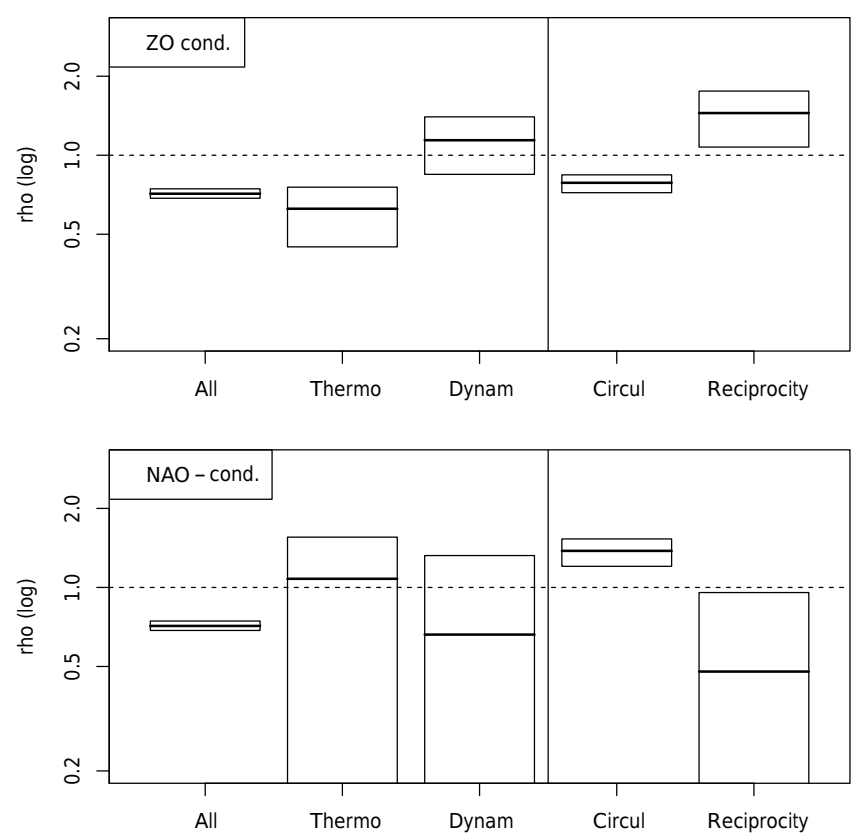

Figure 7. Changes in probability ratios from weather regimes in Weather@Home simulations. The probability ratios (vertical axes) are shown on a logarithmic scale. The horizonal dashed lines show the reference $\rho=1$ line. The dynamical contribution is the product of the circulation and reciprocity contributions. The upper panel is the conditional probability ratios for the zonal regime. The lower panel is for the NAO- regime. The thick horizontal segment represents the estimated ratio $\bar{\rho}$ from all available data. The boxes represent the bootstrap confidence $90 \%$ intervals $\left(\bar{\rho}-\left(\hat{\rho}^{95 \%}-\bar{\rho}\right), \bar{\rho}-\right.$ $\left.\left(\hat{\rho}^{5 \%}-\bar{\rho}\right)\right)$, where $\hat{\rho}^{5 \%}$ and $\hat{\rho}^{95 \%}$ are respectively the 5th and 95 th quantiles of the bootstrap samples.

The NAO - yields a quite different picture, although it can lead to wet winters in southern UK (Fig. 5). The $\rho^{\text {the }}$ ratio is not distinguishable from 1 and has a large variability. Therefore, it cannot be concluded that this weather regime has a significant thermodynamic contribution to changes of heavy precipitation rates. $\bar{\rho}^{\text {circ }}>1$ means that the mean January precipitation rate decreases for NAO- from $\mathcal{W}_{0}$ to $\mathcal{W}_{1}$. The reciprocity ratio $\bar{\rho}^{\text {rec }}$ is lower than 1 , meaning that NAO- is less likely during episodes of high precipitation. This means that the NAO- regime becomes less frequent and less rainy, in contradistinction to the zonal regime.

An analogue-like approach was used to estimate the $\rho$ decomposition from the Weather@Home data. The distance between the January 2014 SLP in NCEP and each Weather@Home simulation was computed, as the average of daily SLP distances. Then the neighborhood of $C_{\text {ref }}=$ $C_{\text {Jan.2014 }}$ is defined when this average distance is lower than a threshold estimated from analogues of NCEP data. The value of the threshold is 1.5 times the average (over January 2014) of the median of the distances of the 20 best daily analogues. This leads to a threshold value of $12 \mathrm{hPa}$ and defines the "circulation tube" of Sect. 3.3.2. In this way, the conditional

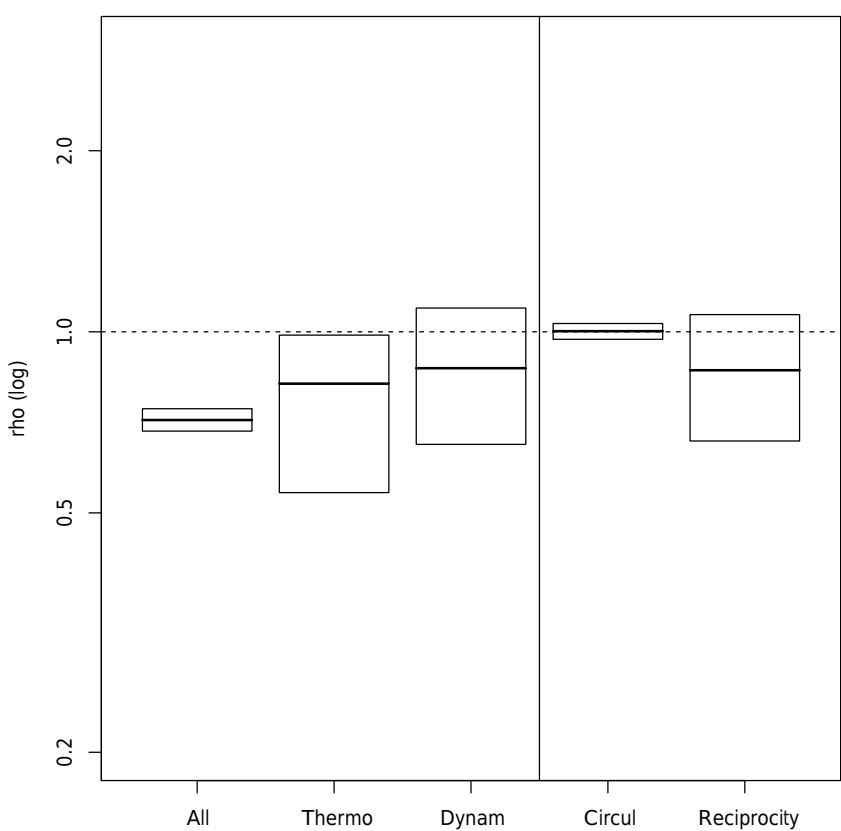

Figure 8. Changes in probability ratios from the analogue approach in Weather@Home simulations. The probability ratios (vertical axes) are shown on a logarithmic scale. The horizonal dashed lines show the reference $\rho=1$ line. The dynamical contribution is the product of the circulation and reciprocity contributions. The boxes yield the same convention as in Fig. 7.

probabilities (and their sampling distributions) can be estimated by bootstrapping. The sampling distribution of each probability ratio are shown in Fig. 8.

We see that the thermodynamical contribution is very similar to the one of the zonal circulation pattern in Fig. 7, but the dynamical contribution has an opposite sign. The circulation contribution is $\approx 1$, indicating that the probability of having a circulation like the one of January 2014 does not change significantly, while the reciprocity term is lowered. Therefore, the frequency of a persisting zonal weather regime increases between the counterfactual and factual worlds, while probability of having a circulation history that is similar to 2014 remains stable. This apparent contradiction is explained by the fact that the circulation of January 2014, although zonal, was rather dissimilar to the usual zonal weather regime. Hence, by tightening the class of event from "high precipitation sum due to zonal weather regime" to "high precipitation sum due to a specific persisting circulation", we change the quantification of a dynamical contribution.

This emphasizes the need of a precise definition of the neighborhood of a circulation trajectory for the conditional attribution exercise. On the one hand, one looks at a persisting zonal circulation in a rather broad sense. On the other hand, one looks at a circulation trajectory that looks like the observation of January 2014, which yielded an atypical zonal pattern (van Oldenborgh et al., 2015). 

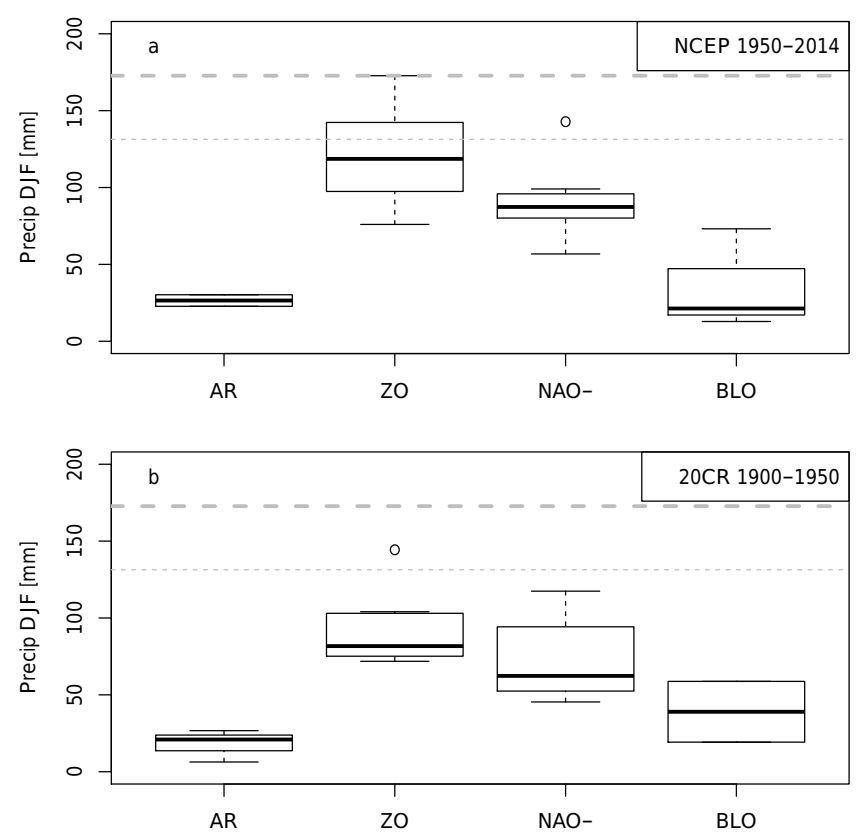

Figure 9. Cumulated southern UK January precipitation (in $\mathrm{mm}$ ) probability distribution conditional to winter weather regimes exceeding $75 \%$ in reanalyses (a: NCEP; b: 20CR). The thin dashed horizontal line is the $99 \%$ quantile of $\mathcal{W}_{1}$ (NCEP). The thick dashed line is the precipitation amount in January 2014.

\subsection{Reanalyses}

The two reanalyses (20CR and NCEP) use different models, assimilation schemes and assimilated data. Schaller et al. (2016, supplementary information) showed that the weather regime classification in the overlapping period of the two reanalyses are very similar. We also verify that the analogues of January 2014 are qualitatively similar in the two reanalyses over the 1950-2011 period. For each day of January 2014, the 20 best analogues have between 12 and 18 days in common in the two reanalyses. The distances and spatial correlation yield probability distributions that cannot be distinguished by a Kolmogorov-Smirnov test (von Storch and Zwiers, 2001).

We set a high threshold of precipitation to the 99th quantile of January cumulated precipitation. Due to the rather low number of data points, we also considered the months of December and February during which high cumulated winter precipitation is likely. This choice can also be justified because the properties of the atmospheric circulation are baroclinic across the winter (Hoskins and James, 2014). We verify that high values of precipitation $R$ can be obtained with more than one weather regime (namely, the zonal and NAOregimes) in Fig. 9. This justifies that the decomposition of Eq. (2) is repeated for these two weather regimes, although it can be anticipated that this threshold cannot be exceeded for $\mathrm{NAO}-$ in $\mathcal{W}_{0}$ in the observations.
Again, the North Atlantic circulation patterns are discriminating for heavy precipitation in southern UK in the observation universe. Hence, we focus on the $\mathrm{ZO}$ and NAO- atmospheric patterns to compute the probability changes.

Similar estimates of $\rho, \rho^{\text {the }}, \rho^{\text {circ }}$ and $\rho^{\text {rec }}$ were computed from the NCEP $\left(\mathcal{W}_{1}\right.$ from 1951 to 2015$)$ and 20CR $\left(\mathcal{W}_{0}\right.$ from 1900 to 1950) reanalyses (Figure 10). The mean ratio $\bar{\rho}$ is $\approx 0.82((0.36 ; 1.37)$ with a $90 \%$ confidence interval $)$, indicating a FAR value of $\approx 0.18$. The distribution of $\rho$ is not significantly different from 1 (although the sampling distribution is skewed towards a lower value) due to the low number of observations, but its range is compatible with the Weather@Home estimate.

The three ratio distributions $\left(\rho^{\text {the }}, \rho^{\text {circ }}\right.$ and $\left.\rho^{\text {rec }}\right)$ were computed for the zonal and NAO- weather regimes (Fig. 10). The values cannot be determined for the thermodynamical and reciprocity terms because the precipitation threshold is not reached or exceeded in $\mathcal{W}_{0}$ during winters dominated by NAO-

The mean value is significantly different from 1 for the zonal regime $\left(\bar{\rho}^{\text {the }} \approx 0.36(0.2,0.71)\right.$ for a $90 \%$ confidence interval). They are not significantly different from 1 for the circulation and reciprocity terms $\bar{\rho}^{\text {circ }} \approx 0.89(0.12,1.34)$ and $\bar{\rho}^{\text {rec }} \approx 2.5(0.2,4)$ ). This description is qualitatively similar to what was obtained with the Weather@Home analysis for the thermodynamical and dynamical terms, although the magnitudes differ, due to the differences between the two universes (factual vs. counterfactual, and new vs. old). The uncertainty increase is partly due to the limited lengths of the reanalysis datasets. The mean reciprocity ratio $\bar{\rho}^{\text {rec }}$ is rather close to what was found in the Weather@Home analysis. It indicates an increase of zonal circulation when heavy precipitation occurs between the beginning of the 20th century and the present-day period.

The $\rho$ ratio distributions for the NAO- regime are not very informative. The thermodynamic and reciprocity contributions cannot be estimated because the threshold of precipitation is never reached during a winter dominated by NAO - in the NCEP reanalysis, between 1951 and 2014, implying zero denominators in Eqs. (3), (5). A first interpretation is that the NAO- regime is so different in both worlds that the conditional precipitation change cannot be estimated (because $\operatorname{Pr}\left(R_{(1)}>R_{\text {ref }} \mid C_{(1)} \in \mathcal{V}\left(C_{\text {ref }}\right)\right)=0$ and $\left.\operatorname{Pr}\left(C_{(1)} \in \mathcal{V}\left(C_{\text {ref }}\right) \mid R_{(1)}>R_{\text {ref }}\right)=0\right)$. This might be due to the low number of winters in the $\mathcal{W}_{0}$ world (i.e., 50 years).

The ratio distributions with the analysis of SLP analogues is shown in Fig. 11. The distribution of $\rho^{\text {the }}$ yields a smaller variance than with the weather regime description due to the tighter constraint on the shape of the atmospheric trajectory. The dynamical term $\rho^{\text {dyn }}$ is barely above 1 (contrary to the $\mathrm{ZO}$ weather regime in the same worlds), although not significantly.

This apparent contradiction is explained by the fact that the $\mathrm{ZO}$ weather regime becomes slightly more probable in $\mathcal{W}_{1}$ than in $\mathcal{W}_{0}$ (circulation term in Fig. 10), but the average 

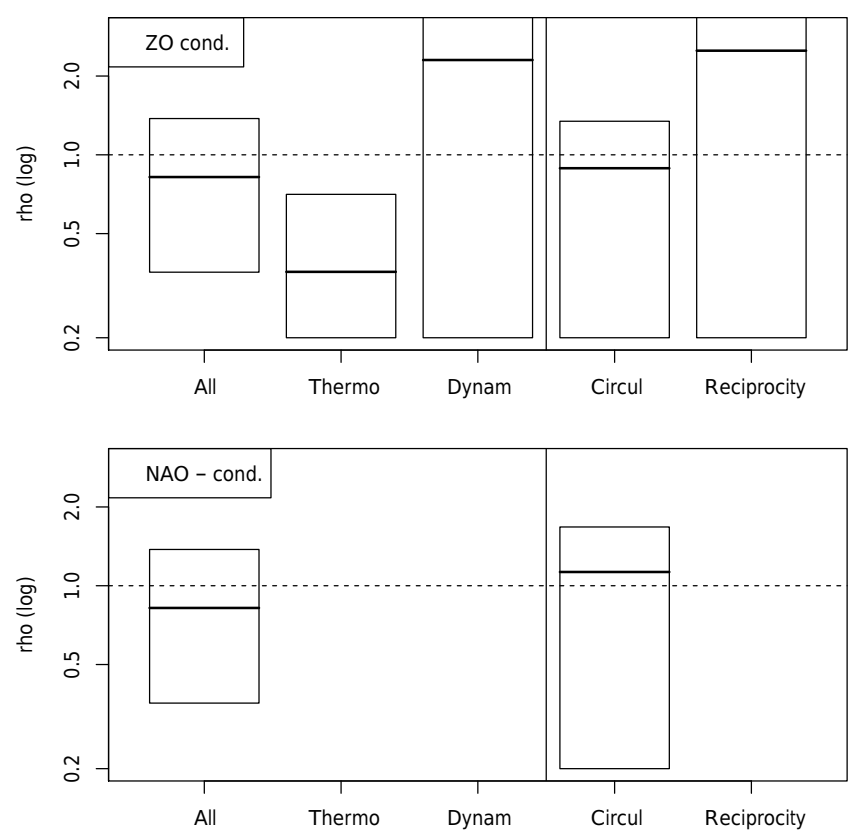

Figure 10. Changes in probability ratios in 20CR/NCEP reanalyses for the zonal and NAO- weather regimes. The probability ratios (vertical axes) are shown on a logarithmic scale. The horizonal dashed lines show the reference $\rho=1$ line. The dynamical contribution is the product of the circulation and reciprocity contributions. The upper panel is the conditional probability ratios for the zonal regime. The lower panel is for the NAO- regime. There are no thermodynamical or reciprocity terms in the decomposition because high precipitation sums do not occur during persisting NAOepisodes in 1900-1950. The boxes yield the same convention as in Fig. 7.

distance of SLP analogues of January 2014 slightly increases between $\mathcal{W}_{0}$ and $\mathcal{W}_{1}$ (Fig. 12). This reflects the fact that the January 2014 pattern is not a typical zonal pattern (as seen in Fig. 3) and that the thermodynamical term outbalances the dynamical term in the interpretation of $\rho<1$.

The analogue method does not allow for an estimate of the circulation and reciprocity terms because we are only able to sample trajectories around January 2014, not all trajectories like in the Weather@Home experiments.

\section{Discussion}

We have performed analyses on two different world definitions (factual vs. counterfactual and new vs. old). There is no quantitative way of claiming that factual equals new and counterfactual equals old. It is only possible to argue qualitatively that the anthropogenic forcings were weaker in the old world than in the new world.

One of the caveats of attribution studies (including this one) is the uncertainty in the $\mathcal{W}_{0}$ world, which affects estimates of $p_{0}$. This problem exists in the counterfactual simulations of Weather@Home, which required the subtraction

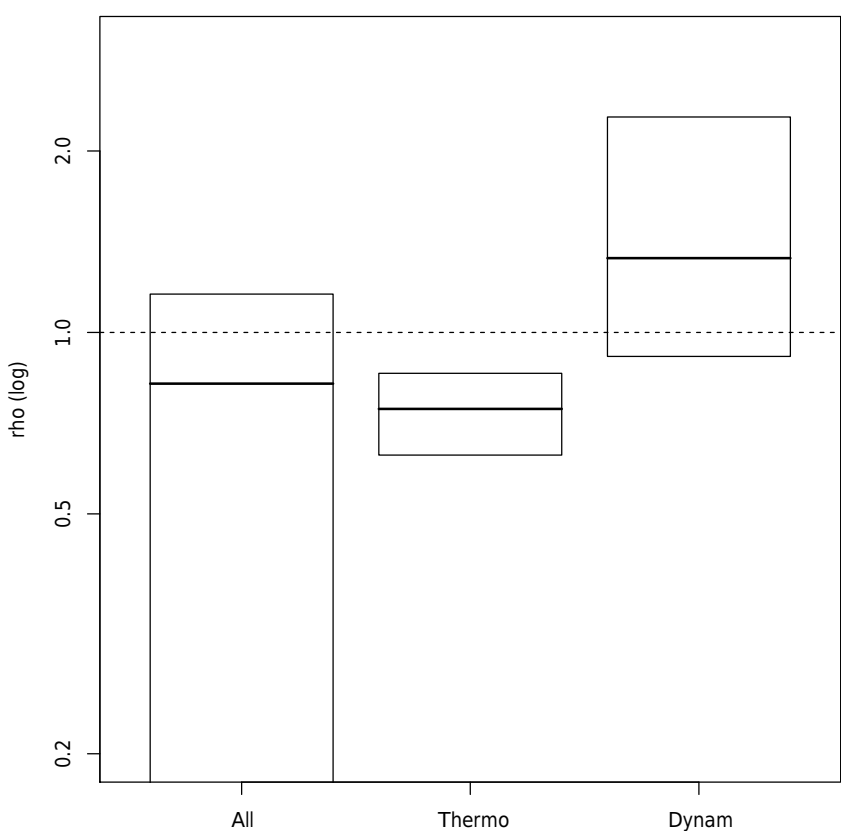

Figure 11. Changes in probabilities in 20CR/NCEP reanalyses conditional to the January 2014 SLP pattern, with circulation analogues. The boxes yield the same convention as in Fig. 7 .

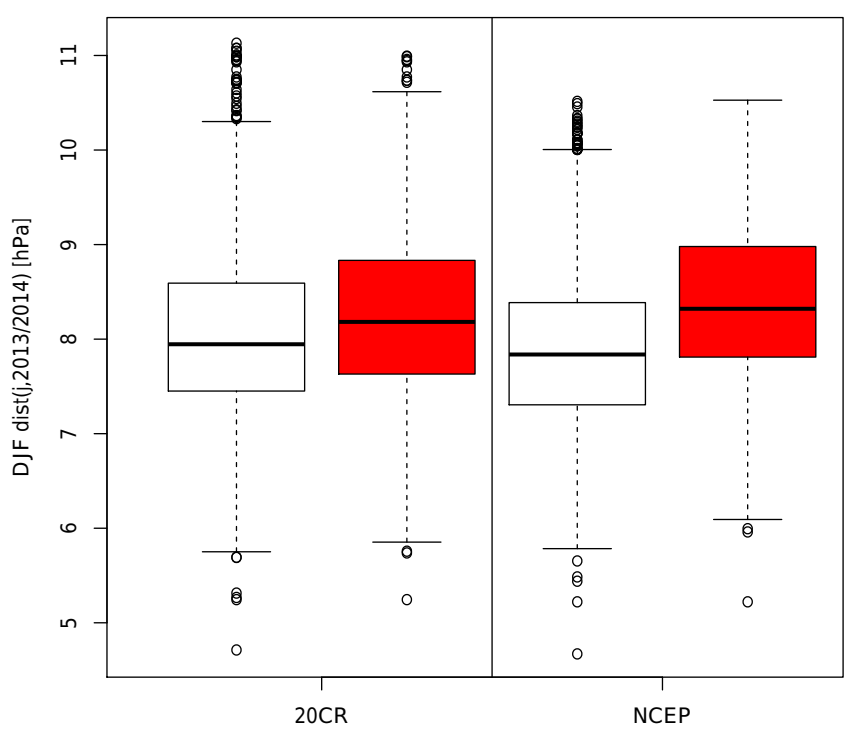

Figure 12. Distribution of mean distances (in hPa) between winter 2013/2014 and the 20 best analogues in NCEP and 20CR. The black box plot are for the whole winter (DJF) and the red box plot are for January 2014 only.

of an SST signal from 11 available CMIP5 simulations. Each of the individual counterfactual simulations show different behavior, although the ensemble yields a significant, albeit small, change with respect to $\mathcal{W}_{1}$, as shown by Schaller et al. (2016). The quality and quantity of the data that were used in the reanalysis experiments varies with time. This implies 
that the old world is more uncertain that the new world. The distributions of distances between analogues in Fig. 12 do not show large systematic biases in 20CR (1900-1950) with respect to NCEP (1951-2014). Using the whole ensemble of 20CR could allow for better estimates of weather regime frequency distributions in the $\mathcal{W}_{0}$ world, but the only precipitation data we used come from observations, which means that uncertainties in the $\rho$ ratio are always large. Another possibility is to consider subperiods of 1900-1950, but the confidence for individual subperiods is bound to be very poor.

The analysis does not consider internal temporal variability in each world. The Weather@Home simulations do not have decadal variability, but reanalyses do. This was not taken into account here, but could be included by further dividing the two worlds (old vs. new) into subperiods (e.g., "high SST" vs. "low SST") in order to evaluate the feedback of natural SST variability on atmospheric circulation. This poses the problem of the length of available data onto which the statistics are built. This difficulty could be overcome by investigating ensembles of available simulations such as CMIP5 (Taylor et al., 2012) or CORDEX (Jacob et al., 2013).

The main assumption made in the Bayes decomposition is that the climate variable $R$ is related to the atmospheric circulation field $C$, and that a storyline of $C$ can explain an observed extreme of $R$. This ensures that the two conditional probabilities in Eq. (2) are non-zero so that the ratios are well defined.

In order to provide consistent results, it is necessary to have a correct representation of the atmospheric variability. This assumption is not trivial and required many verifications on the Hadley Center atmospheric model (Schaller et al., 2016). The circulation patterns that were simulated were validated over the North Atlantic region and Europe for the $\mathcal{W}_{1}$ factual world. The main difficulty is that there is no way to assess the validity of $C$ in the $\mathcal{W}_{0}$ counterfactual world. This is where the assumption that $\mathcal{W}_{1}$ and $\mathcal{W}_{0}$ are close to each other is heuristically used in the estimate of the probability changes. Of course, this is not a strict proof of validation of the atmospheric circulation in $\mathcal{W}_{0}$.

When reanalysis data are used, the question of the atmospheric circulation validity and the $R-C$ relation is tied to the quality of the data that are used in the assimilation scheme, for both worlds $\mathcal{W}_{0}$ and $\mathcal{W}_{1}$. The main caveat is that the early period of reanalyses are constrained by only a few observations (Compo et al., 2011). This means that the circulation reconstruction could yield wrong patterns (even for the members of the ensemble), with no possible validation test. The second caveat in this case is the length of datasets on which the probabilities are computed. Moreover, the observed climate (or its reanalysis) is one occurrence of many possible realizations that could have happened for a given climatic state. Therefore, this analysis should also be understood as being conditional to a dataset (either Weather@Home or the earlier part of the 20CR reanalysis), which is an uncertain representation of the world.
Our paper outlined an apparent discrepancy between weather regime and analogues of circulation to describe thermodynamical changes (and dynamical ones). Weather regimes offer a rather rough description of the atmospheric flow and the range of possible flows within a weather regime classification can be fairly large. The recent winter of 2015/2016 demands a finer description of the atmospheric circulation. Indeed, December 2015 had a mostly zonal weather regime (such as January 2014), with very mild temperatures in Europe, but southern UK and northwestern France were very dry (such as the rest of continental Europe), whereas northern UK experienced record precipitation and floods. The jet stream was slightly shifted (a few hundred kilometers) to the north, but the weather regime was still zonal, while having no resemblance to January 2014 (in terms of analogues). This questions the focus of extreme event attribution on regional climate precipitation alone, as already discussed by Trenberth et al. (2015), since the largescale atmospheric circulation that drives the moisture transport can have shifts within the same weather regime and hit a region rather than its neighbors just by chance. This suggests an EEA analysis of the predictands of $R$ (such as $C$ ), rather than $R$ alone, with a focus on the dynamical terms.

Vautard et al. (2016) proposed an alternative method based on analogues to determine dynamical and thermodynamical components from the Weather@Home simulation data. It is interesting to notice that there is a consensus on the estimate of a thermodynamical term (i.e., with equal atmospheric circulation). Our finding emphasizes that a definition of a dynamical contribution is potentially ambiguous. We also emphasize that the approach of analogues can also be applied to daily Weather@Home data (Fig. 8). Vautard et al. (2016) investigated all possible patterns of atmospheric circulation on a monthly timescale, while this study focuses on January 2014 , with a daily timescale.

The persistence of events and hence the timescale to be considered are major components to be considered. For instance, the probabilities of having a persistent zonal weather regime during a month and having a circulation that is similar to January 2014 have different distributions, and such distributions change in different ways between the two reanalysis datasets. Such a consideration is crucial for regional climate studies; as mentioned above, the example we chose in this paper is about precipitation in southern UK (and arguably northwestern France, which also had records of precipitation in January 2014). But case studies such as northern UK (in December 2015) or Wales in 2000 (Pall et al., 2011) would require separate analyses because the difference in atmospheric flows is different in a subtle but crucial way.

It is desirable to be systematic in the attribution of extreme events in continuous time, by examining all events. This pleads for analyses that can be performed quickly in order to estimate statistical diagnostics in a relatively short time. This can help guide the choice of costly experiments 
(in terms of computing power and memory management), such as Weather@Home, in order to refine estimates.

\section{Conclusions}

We have argued that the use of relatively short datasets (reanalyses) provides qualitatively similar information in terms of probability decomposition of the occurrence of a winter flood event. Such an analysis cannot replace Weather@Home simulations in order to quantify precisely the contribution of all factors. Therefore, the exercise with reanalyses is a detection rather than a thorough attribution, as defined by Bindoff et al. (2013). The attribution comes if the forcing changes are clearly identified in both periods, which is not done in this paper.

The names of terms (thermodynamical and dynamical) of the decomposition can be debated. It is important to note that changes in the properties of the atmospheric circulation $C$ and the coupling between the local climate variables $R$ and $C$ play an important role in the definition of the extreme event.

The conditional part of the analysis is the most important point as it helps to explore the tail of the distribution of $R$. We emphasize that we analyze a high precipitation rate $\left(R>R_{\text {ref }}\right)$ conditional to a given circulation pattern $C_{\text {ref. }}$. We had to make the analysis of the two types of weather regimes leading to high precipitation rates. The thermodynamical and dynamical contributions differed from one weather regime to the other. We also showed that the dynamical contribution to $\rho$ depends on the way the neighborhood of the circulation trajectory is approximated (qualitative with weather regimes or quantitative with analogues). This points to the necessity of an a priori definition of the class events to be investigated, in order to obtain consistent results when following a storyline approach to extreme event attribution.

We emphasize that the paradigm of attribution of extreme events that we have explored can also be applied to other contexts, in particular extreme events of the last millennium as a response to solar and volcanic forcings (Schmidt et al., 2011, 2014; PAGES 2k-PMIP3 group, 2015). This can be done by exploring analogues of circulation of a given extreme event in remote periods (in model simulations) where natural forcings are well documented.

Data availability. NCEP reanalysis data can be obtained from the NOAA web site (https://www.esrl.noaa.gov/psd/data/gridded/ data.ncep.reanalysis.html). Weather@Home data can be obtained upon request (cpdn@oerc.ox.ac.uk). Southern UK precipitation data were obtained from the UK Met Office (Tim Legg, tim.legg @ metoffice.gov.uk).

Competing interests. The authors declare that they have no conflict of interest.
Acknowledgements. It is a pleasure to thank Ted Shepherd (U Reading) for useful discussions on the Bayesian decomposition. The manuscript benefited from the constructive comments of three anonymous referees. P. Yiou is supported by the ERC grant no. 338965-A2C2. This work is also supported by the Copernicus EUCLEIA project no. 607085.

Edited by: M. Wehner

Reviewed by: three anonymous referees

\section{References}

Allen, M.: Liability for climate change, Nature, 421, 891-892, 2003.

Bindoff, N., Stott, P., AchutaRao, K., Allen, M., Gillett, N., Gutzler, D., Hansingo, K., Hegerl, G., Hu, Y., Jain, S., Mokhov, I., Overland, J., Perlwitz, J., Sebbari, R., and Zhang, X.: Detection and Attribution of Climate Change: from Global to Regional, Cambridge University Press, Cambridge, United Kingdom and New York, NY, USA, 867-952, 2013.

Cattiaux, J., Vautard, R., Cassou, C., Yiou, P., Masson-Delmotte, V., and Codron, F.: Winter 2010 in Europe: A cold extreme in a warming climate, Geophys. Res. Lett., 37, L20704, doi:10.1029/2010g1044613, 2010.

Christidis, N. and Stott, P.: Extreme rainfall in the United Kingdom during winter 2013/14: The role of atmospheric circulation and climate change, B. Am. Meteorol. Soc., 96, S46-S50, doi:10.1175/BAMS-D-15-00094.1, 2015.

Compo, G., Whitaker, J., Sardeshmukh, P., Matsui, N., Allan, R., Yin, X., Gleason, B., Vose, R., Rutledge, G., Bessemoulin, P., Brünnimann, S., Brunet, M., Crouthamel, R., Grant, A., Groisman, P., Jones, P., Kruk, M., Kruger, A., Marshall, G., Maugeri, M., Mok, H., Nordli, O., Ross, T., Trigo, R., Wang, X., Woodruff, S., and Worley, S.: The Twentieth Century Reanalysis Project, Q. J. Roy. Meteorol. Soc., 137, 1-28, doi:10.1002/qj.776, 2011.

Hannart, A., Pearl, J., Otto, F., Naveau, P., and Ghil, M.: Causal counterfactual theory for the attribution of weather and climaterelated events, B. Am. Meteorol. Soc., 97, 99-110, 2016.

Hoskins, B. J. and James, I. N.: Fluid Dynamics of the Mid-Latitude Atmosphere, J. Wiley \& Sons, 2014.

Huntingford, C., Marsh, T., Scaife, A. A., Kendon, E. J., Hannaford, J., Kay, A. L., Lockwood, M., Prudhomme, C., Reynard, N. S., Parry, S., Lowe, J. A., Screen, J. A., Ward, H. C., Roberts, M., Stott, P. A., Bell, V. A., Bailey, M., Jenkins, A., Legg, T., Otto, F. E. L., Massey, N., Schaller, N., Slingo, J., and Allen, M. R.: Potential influences on the United Kingdom's floods of winter 2013/14, Nature Clim. Change, 4, 769-777, 2014.

Jacob, D., Petersen, J., Eggert, B., Alias, A., Christensen, O. B., Bouwer, L. M., Braun, A., Colette, A., Déqué, M., Georgievski, G., Georgopoulou, E., Gobiet, A., Menut, L., Nikulin, G., Haensler, A., Hempelmann, N., Jones, C., Keuler, K., Kovats, S., Kroner, N., Kotlarski, S., Kriegsmann, A., Martin, E., Meijgaard, E., Moseley, C., Pfeifer, S., Preuschmann, S., Radermacher, C., Radtke, K., Rechid, D., Rounsevell, M., Samuelsson, P., Somot, S., Soussana, J.-F., Teichmann, C., Valentini, R., Vautard, R., Weber, B., and Yiou, P.: EURO-CORDEX: new high-resolution climate change projections for European impact research, Reg. Environ. Change, 14, 563-578, doi:10.1007/s10113-013-0499-2, 2013. 
Kalnay, E., Kanamitsu, M., Kistler, R., Collins, W., Deaven, D., Gandin, L., Iredell, M., Saha, S., White, G., Woollen, J., Zhu, Y., Chelliah, M., Ebisuzaki, W., Higgins, W., Janowiak, J., Mo, K., Ropelewski, C., Wang, J., Leetmaa, A., Reynolds, R., Jenne, R., and Joseph, D.: The NCEP/NCAR 40-year reanalysis project, B. Am. Meteorol. Soc., 77, 437-471, 1996.

Kimoto, M. and Ghil, M.: Multiple flow regimes in the Northernhemisphere winter. 1. Methodology and hemispheric regimes, J. Atmos. Sci., 50, 2625-2643, 1993.

Massey, N., Jones, R., Otto, F. E. L., Aina, T., Wilson, S., Murphy, J. M., Hassell, D., Yamazaki, Y. H., and Allen, M. R.: weather@home development and validation of a very large ensemble modelling system for probabilistic event attribution, Q. J. Roy. Meteor. Soc., 141, 1528-1545, doi:10.1002/qj.2455, 2015.

Matthews, T., Murphy, C., Wilby, R. L., and Harrigan, S.: Stormiest winter on record for Ireland and UK, Nature Clim. Change, 4, 738-740, doi:10.1038/nclimate2336, 2014.

Michelangeli, P., Vautard, R., and Legras, B.: Weather regimes: Recurrence and quasi-stationarity, J. Atmos. Sci., 52, 1237-1256, 1995.

National Academies of Sciences Engineering and Medicine: Attribution of Extreme Weather Events in the Context of Climate Change, The National Academies Press, Washington, DC, doi:10.17226/21852, 2016.

PAGES 2k-PMIP3 group: Continental-scale temperature variability in PMIP3 simulations and PAGES 2k regional temperature reconstructions over the past millennium, Clim. Past, 11, 16731699, doi:10.5194/cp-11-1673-2015, 2015.

Pall, P., Aina, T., Stone, D. A., Stott, P. A., Nozawa, T., Hilberts, A. G. J., Lohmann, D., and Allen, M. R.: Anthropogenic greenhouse gas contribution to flood risk in England and Wales in autumn 2000, Nature, 470, 382-385, doi:10.1038/Nature09762, 2011.

Pearl, J.: Causality, Cambridge Univ. Press, 2009.

Peixoto, J. P. and Oort, A. H.: Physics of climate, American Institute of Physics, New York, 1992.

Schaller, N., Kay, A. L., Lamb, R., Massey, N. R., van Oldenborgh, G. J., Otto, F. E. L., Sparrow, S. N., Vautard, R., Yiou, P., Ashpole, I., Bowery, A., Crooks, S. M., Haustein, K., Huntingford, C., Ingram, W. J., Jones, R. G., Legg, T., Miller, J., Skeggs, J., Wallom, D., Weisheimer, A., Wilson, S., Stott, P. A., and Allen, M. R.: Human influence on climate in the 2014 southern England winter floods and their impacts, Nature Clim. Change, 6, 627-634, doi:10.1038/nclimate2927, 2016.

Schmidt, G. A., Jungclaus, J. H., Ammann, C. M., Bard, E., Braconnot, P., Crowley, T. J., Delaygue, G., Joos, F., Krivova, N. A., Muscheler, R., Otto-Bliesner, B. L., Pongratz, J., Shindell, D. T., Solanki, S. K., Steinhilber, F., and Vieira, L. E. A.: Climate forcing reconstructions for use in PMIP simulations of the last millennium (v1.0), Geosci. Model Dev., 4, 33-45, doi:10.5194/gmd4-33-2011, 2011.

Schmidt, G. A., Annan, J. D., Bartlein, P. J., Cook, B. I., Guilyardi, E., Hargreaves, J. C., Harrison, S. P., Kageyama, M., LeGrande, A. N., Konecky, B., Lovejoy, S., Mann, M. E., Masson-Delmotte, V., Risi, C., Thompson, D., Timmermann, A., Tremblay, L.B., and Yiou, P.: Using palaeo-climate comparisons to constrain future projections in CMIP5, Clim. Past, 10, 221-250, doi:10.5194/cp-10-221-2014, 2014.
Shepherd, T. G.: A Common Framework for Approaches to Extreme Event Attribution, Current Climate Change Reports, 2, 28 38, doi:10.1007/s40641-016-0033-y, 2016.

Stott, P. A., Stone, D. A., and Allen, M. R.: Human contribution to the European heatwave of 2003, Nature, 432, 610-614, doi:10.1038/Nature03089, 2004.

Taylor, K. E., Stouffer, R. J., and Meehl, G. A.: An Overview of CMIP5 and the Experiment Design, B. Am. Meteorol. Soc., 93, 485-498, 2012.

Trenberth, K. E., Fasullo, J. T., and Shepherd, T. G.: Attribution of climate extreme events, Nature Clim. Change, 5, 725-730, doi:10.1038/nclimate2657, 2015.

van Haren, R., van Oldenborgh, G. J., Lenderink, G., and Hazeleger, W.: Evaluation of modeled changes in extreme precipitation in Europe and the Rhine basin, Envir. Res. Lett., 8, 014053, doi:10.1088/1748-9326/8/1/014053, 2013.

van Oldenborgh, G. J., Stephenson, D. B., Sterl, A., Vautard, R., Yiou, P., Drijfhout, S. S., von Storch, H., and van den Dool, H.: Drivers of the 2013/14 winter floods in the UK, Nature Clim. Change, 5, 490-491, doi:10.1038/nclimate2612, 2015.

Vautard, R., Legras, B., and Déqué, M.: On the source of midlatitude low-frequency variability. 1. A Statistical approach to persistence, J. Atmos. Sci., 45, 2811-2843, 1988.

Vautard, R., Yiou, P., Otto, F., Stott, P., Christidis, N., van Oldenborgh, G. J., and Schaller, N.: Attribution of human-induced dynamical and thermodynamical contributions in extreme weather events, Environ. Res. Lett., 11, 114009, doi:10.1088/17489326/11/11/114009, 2016.

von Storch, H. and Zwiers, F. W.: Statistical Analysis in Climate Research, Cambridge University Press, Cambridge, 2001.

Yiou, P.: AnaWEGE: a weather generator based on analogues of atmospheric circulation, Geosci. Model Dev., 7, 531-543, doi:10.5194/gmd-7-531-2014, 2014.

Yiou, P. and Nogaj, M.: Extreme climatic events and weather regimes over the North Atlantic: When and where?, Geophys. Res. Lett., 31, L07202, doi:10.1029/2003GL019119, 2004.

Yiou, P., Goubanova, K., Li, Z. X., and Nogaj, M.: Weather regime dependence of extreme value statistics for summer temperature and precipitation, Nonlin. Processes Geophys., 15, 365-378, doi:10.5194/npg-15-365-2008, 2008.

Yiou, P., Salameh, T., Drobinski, P., Menut, L., Vautard, R., and Vrac, M.: Ensemble reconstruction of the atmospheric column from surface pressure using analogues, Clim. Dynam., 41, 13331344, doi:10.1007/s00382-012-1626-3, 2013. 